\title{
ABtTCE Models of care in forensic psychiatry
}

\author{
Harry G. Kennedy (1)
}

Harry G. Kennedy, BSc, MB, MD, FRCPI, FRCPsych, is a Clinical

Professor of Forensic Psychiatry at Trinity College Dublin, and Executive Clinical Director of the National Forensic Mental Health Service, Central Mental Hospital, Dublin, Ireland. He is also Visiting Skou Professor of Forensic Psychiatry at Aarhus University, Denmark. Qualified in medicine in Dublin and trained in medicine (Royal Postgraduate Medical School) and forensic psychiatry (Maudsley Hospital and Institute of Psychiatry) in London, he worked as consultant forensic psychiatrist and clinical director in North London before returning to his present position in 2000. Professor Kennedy is involved in planning and implementing the move of Dublin's Central Mental Hospital from an 1850s' building to a new purpose-built secure forensic hospital campus. He teaches forensic psychiatry and publishes research on the epidemiology of homicide and suicide, triage and recovery in therapeutically safe and secure pathways (the DUNDRUM toolkit), forensic models of care, neuroscience and violence, mental health law and human rights.

Correspondence Harry G. Kennedy. Email: kennedh@tcd.ie

First received 12 Jan 2021

Final revision 24 Mar 2021

Accepted 30 Mar 2021

\section{Copyright and usage}

(C) The Author(s), 2021. Published by Cambridge University Press on behalf of the Royal College of Psychiatrists. This is an Open Access article, distributed under the terms of the Creative Commons AttributionNonCommercial-NoDerivatives licence (http://creativecommons.org/ licenses/by-nc-nd/4.0/), which permits non-commercial re-use distribution, and reproduction in any medium, provided the original work is unaltered and is properly cited. The written permission of Cambridge University Press must be obtained for commercial re-use or in order to create a derivative work.

\section{SUMMARY}

Forensic psychiatry services have grown and become more complex in structures, processes and pathways. Legacy customs, practices and changing policy are now organised into formal models of care. These are written accounts of how a health service is delivered, outlining best practice and services for patients progressing through the stages of their condition and the care and treatment available. This article explores the four key elements of a model of care: goals; pathways and processes; treatment programmes; and systematic evaluation. It describes the most common model of care in forensic services, which builds on structures of stratified therapeutic security. It also considers variations on this basic or standard model matched to needs arising from the complex interrelationship with other parts of the mental health service for the population served and with criminal justice, primary care and physical health, housing and welfare agencies.

\section{LEARNING OBJECTIVES}

After reading this article you will be able to:

- understand what a model of care is and how it contributes to the running of a forensic mental health service

- participate in the design and drafting of a model of care for a forensic psychiatry service

- evaluate a service and compare service models.

\section{KEYWORDS}

Forensic psychiatry; clinical science; mental health; psychiatric services; relational therapeutic security.

A model of care is a document that is intended to be read by all new staff joining a healthcare service. The model of care bears the same relationship to policies and operational procedures that a constitution bears to laws and statutory instruments. Forensic mental health services are designed to deliver effective care and treatment for people with severe mental disorders who for a time are a danger to others. Dangerousness arises from concern about a combination of risk (probability) and seriousness of harm. Forensic patients have a right to equal access to treatment even during periods when delivery of that treatment requires a therapeutically safe and secure setting. Forensic mental health services for mentally disordered offenders and those like them are distinguished from other mental health services by a dual mandate to act in the best interests of the patient and in the public interest. This often involves providing care and treatment in conditions of therapeutic security, so that patients and clinicians are safe while treatment is provided. Forensic mental health services are integral parts of the larger mental health services for the population they serve, part of an interdependent system (Gunn 1977; O'Grady 1990). Any change in the delivery of care in one part of the overall mental health service will have effects on all the other parts (Kennedy 2002; O’Reilly 2019b). Large systems change and re-equilibrate slowly. A whole systems approach is always necessary when understanding the working of a model of care. Changes in mental health and criminal justice policy can also be expected to have large effects on services.

A model of care is not the same thing as a nursing model (actually a nursing process) or the medical model (actually conceptual, scientific and heuristic approaches to diagnosis, causation and treatment with closer resemblance to a culture of expertise). The modern idea of a model of care owes much to ideas taken from systems theory, including the interdependence of parts, the mathematical modelling of stable states in closed systems that include servofeedback loops and the complications of open and unstable systems (Von Bertalanffy 1969).

A model of care broadly defines the way health services are delivered. It outlines best practice and services for a person, population group or patient cohort as they progress through the stages of the condition, injury or event. It aims to ensure that people get the right care, at the right time, by the right team and in the right place. A model of care often includes a logic model relating inputs (resources and time) to outputs (health gains). This definition is derived from the New South Wales Agency for Clinical Innovation (Agency for Clinical Innovation 2013). A model of care should be written in plain English. However, it is not the same as a prospectus for patients or their families. Nor is it a contracting document. A model of care should be designed to last without major modification for about 5 years so that it can be evaluated 
properly. A model of care is typically written for the first time in consultation with commissioners, in order to instruct architects, software and clinical management system designers or as part of service reform. Although a model of care cannot in itself cause a service to achieve its goals, it may be a precondition for success and it can contribute to a shared vision, mission and values.

A model of care can be written or compiled in many ways. Where no pre-existing written model of care can be identified, a starting point is to identify the existing tacit or implicit model of care by mapping existing services, their estate, human resources and levels of activity, pathways and processes. A common approach is to engage stakeholders in a process of participatory action research. This has been described as a spiral of steps each of which consists of planning, action and evaluation of the result of the action. Central to this is the involvement of all stakeholders (Giblin 2012). However, a model of care for a forensic psychiatry service cannot be written in a coherent way from grass roots up. A vision is necessary (Gunn 1977). A model of care should be derived from clinical science that is consistent, methodical, cumulative and capable of being evaluated objectively. Human rights, medical ethics, legal structures and processes are 'given'. Legal structures and processes will shape service demands and service processes. A final version of the model of care can only be valid if accepted by the clinical experts who have clinical responsibility.

Although the definition above is intended to be generic and applies to any health service or part of a service, the emphasis on relating needs to levels of service is close in meaning to the risk-needsresponsivity model adopted for forensic mental health services.

\section{Elements of a model of care}

The key elements of a model of care are:

- goals (not principles)

- pathways and processes

- treatments

- evaluation and logic models.

I will discuss each in turn.

\section{Goals}

Principles are typically derived from international conventions on the rights of patients, constitutional rights and legislation. Lists of principles are seldom difficult to agree on, although the ordering and prioritising of principles can be very difficult. Good ideas are never good enough - evidence for effectiveness must be shown and weighed against unintended consequences (Duggan 2011; Gibbons
BOX 1 Goals for forensic mental health services

- Rights and recovery as defined in legal standards

- Zero target for violence by patients against patients and others

- Prioritisation of effective treatments over any other activity

- Active management of length of stay

- Population-based levels of service that are sustainable

2015) or banishment pressure, the exclusion of difficult patients who do not fit a theory or model of care (Gunn 1977). Goals are therefore the most useful starting point for a model of care (Box 1). Goals are derived from principles, but goals are easier to prioritise and to define in ways that are measurable and achievable. Falk set out basic goals for secure services: (a) sufficient physical security appropriate to the patients; (b) high staff:patient ratios; and (c) a therapeutic policy that encompasses individual programmes (Falk 1985).

An emphasis should be placed on quality and excellence. Quality standards ensure that a floor is set below which the service will not fall, with a cyclical process for continuous service improvement to meet those standards (Aimola 2016). Excellence standards ensure continuously improving outcomes for patients through consistency, governance and integrated networks between services for research, development, teaching and training (Kennedy 2019a). Because forensic mental health services are high risk, low volume and high cost, quality standards are essential as a grounding, and demonstrable excellence is a necessary part of ensuring a resilient service that can cope with adverse events.

\section{Pathways and processes}

Forensic mental health services include a secure forensic hospital at their core. These function as specialist tertiary referral centres. A hospital is usually distinguished from a prison by medical governance rather than having a prison governor as the detaining authority. Typically, secure forensic hospitals and forensic mental health services sit at the centre of a complex intersection of pathways from the criminal justice system and prisons and from second tier specialist psychiatric services, with complex pathways back to the community involving many agencies (Box 2).

A population-based forensic mental health service must also include court liaison and diversion services, psychiatric in-reach clinics in remand and sentenced prisons, community aftercare and 
BOX 2 Processes and structures

- Clinical processes occur along all forensic pathways, including triage, leave, transfer to less secure places, trial leave, conditional discharge and absolute discharge.

- Active management of length of stay through admission panels and further 'gating' panels for decisions regarding milestones of progress.

- Structured professional judgement and judgement-support frameworks for making governance decisions regarding clinical processes.

- Legal processes to facilitate goals through clinical processes, including medical necessity, and dual mandate.

supervision services as well as consultation and liaison services for general adult mental health services and criminal justice agencies. Where these are not provided within one organisation, there should be clearly defined processes for triage, allocation and waiting-list management to ensure that pathways function quickly and responsively according to need. Triage criteria should be patient focused rather than institutional and should be described in meaningful units of difference or reliable change.

\section{The elements of therapeutic security}

Secure forensic hospitals and services can always be described in terms of the elements of therapeutic security (Kennedy 2002): environmental or physical security; relational security (quantitative and qualitative); procedural security; and the specialist management and governance arrangements necessary to facilitate and sustain these (Box 3).

\section{Environmental or physical security}

Environmental therapeutic security includes the designed and built environment, maintenance of estate and fittings and the staff necessary to

BOX 3 The elements of therapeutic security

- Environmental or physical

- Relational - quantitative and qualitative

- Procedural

- Management and governance

Each element is stratified from high secure and intensive care, to medium secure, to low secure, to community supports (also stratified).

The elements are also stratified from acute to subacute, and medium term to slow stream or long term.

(Kennedy 2002) operate them. It includes perimeter security, secure entrances and exits, ligature-free environments, clear sight lines and many other design essentials. The environment should be so robust that it can withstand attempts at destruction and cannot be used to produce weapons. At medium and high secure levels the environment should be escape proof. The built environment should also be clean, constantly well maintained, with natural daylight and well-circulated air, and should allow patients some control over their own environment, for example heating and light. It should be pleasing to the eye and uplifting in design and presentation. Although robust and secure, the built environment should not have obvious custodial, penal or nontherapeutic qualities. Access to gardens, vistas and variety (bedrooms, day rooms, therapy areas, classrooms, workshops, gyms) should be designed and planned (Seppanen 2018). There should also be some form of engagement between the management of the secure hospital and the local community.

\section{Relational security}

Relational therapeutic security can be divided into quantitative and qualitative. Quantitative relational therapeutic security starts with the ratio of staff to patients at ward level and the amount of time spent in face-to-face contact. It includes skills mix and the level and sophistication of training and experience. Qualitative relational therapeutic security is the balance between intrusiveness and openness; trust and safe boundaries between patients, their families and professionals; and getting to know patients well, which includes the depth and breadth of knowledge the professionals have about their patients. The ability to maintain a therapeutic relationship with a good working alliance and interpersonal trust, while at the same time systematically managing boundaries so that risk is recognised and managed is essential. This represents one of the higher aspects of forensic psychiatric specialist training and expertise in each of the mental health professions (James 1990; Tighe 2012).

\section{Procedural security}

Procedural therapeutic security serves the first two elements by ensuring that risk assessment and risk management (risk reduction and risk mitigation) are systematic, consistent across the service, tailored to individual need and dynamic. It includes policies and practices for controlling risk. At the patient level this includes systems and routines for the control and checking of patients' movements and communication generally. At the systems level it includes arrangements for professional governance, risk management, consistency in decision-making, 
crisis and contingency planning, formalised reviews and transfer of responsibilities. There should be a system to supplement individual risk assessments with the systemic collation of risk and other information, to prevent bullying of patients by patients, to prevent boundary breaches and to ensure that contraband (drugs, weapons, means of escape or illicit media) is not brought into the hospital or circulated within it. A legal instrument may be required for fairness and justice.

\section{Specialist management and governance}

Management and governance structures and processes should be organised to maintain the elements of therapeutic security according to quality standards and excellence, compliance with national and international standards, sustainability over time and resilience. This should include clarity regarding management of resources, lines of reporting and responsibility. There should be processes for the weekly monitoring and benchmarking of admission, transfer and discharge decisions according to predetermined criteria. There should be processes for ensuring compliance with legal and policy requirements. There should be maintenance of inter-agency relationships and boundaries.

Incident reporting and collation with other forms of information relevant to risks and threats should be systematised for risk management, therapeutic security and organisational learning. Formal and systemic assessment of risks relevant to decisions about levels of leave and privileges is central to the prevention of absconding and contraband, while at the same time ensuring that therapeutic risks are taken to achieve timely movement to less secure placements. Particular care is required regarding child visits.

Resilience in a forensic model of care is the ability to cope with new demands, opportunities and difficulties through planning and preparation, to recover from setbacks and serious adverse events (homicides, riots, hostage incidents, arson, absconding and coordinated escapes, boundary breaches, epidemic outbreaks, surges in demand for services). One formulation identifies four elements of organisational resilience as preventive control (defensive consistency), mindful action (defensive flexibility), performance optimisation (progressive consistency) and adaptive innovation (progressive flexibility) (Denyer 2017). Resilience in a clinical service is closely aligned to the concept of excellence, since it relies on prioritising training, practice and innovation. Resilience requires the ability to respond quickly to new and unanticipated challenges, for example the COVID-19 pandemic (Lemieux 2020), by adopting practices from international experience, by innovation and improvisation, using continuous evaluation and design principles and working flexibly and responsively. Expert medical and clinical leadership is essential for such resilience and innovation and to motivate teams for such flexible working. Professional, non-clinical management is necessary to support clinical leadership.

\section{The standard model}

Secure forensic hospitals can be organised according to principles of therapeutic security in a variety of ways. Very small services have relatively little choice in how they are organised; larger services can have more choice; and a minimum size may be necessary for critical mass to enable the delivery of specialist treatment programmes. The most common design is stratified therapeutic security, in which distinctions are made between high security, medium security and low security (Fig. 1). This allows an efficient allocation of human resources so that there are high staff: patient ratios (high quantitative relational therapeutic security) in acute, subacute and intensive care wards, with progressively lower ratios in medium secure and pre-discharge wards. This system of stratification of risk and matching relational security (Müller-Isberner 2007; Pillay 2008) may intersect with stratification of ward security, from acute and subacute wards, to medium-term medium secure and slow-stream secure or long-term wards and finally to rehabilitation and pre-discharge wards (see 'Pathways governance and management' below). Special considerations arise concerning the provision of secure forensic services for women, for adolescents and for special needs groups.

\section{Sustainable levels of service}

Any forensic hospital, service or system is liable to silt up if the numbers discharged in a given time period cannot keep pace with demand for admissions. This can be expressed in various ways. Bed occupancy should generally be managed at about $85 \%$ to cope with seasonal or other surges in demand. In practice many secure forensic hospitals run at $100 \%$ occupancy. Occupancy above 100\% will reduce relational therapeutic security and is unsafe. Average length of stay in a ward or a hospital can be calculated in various ways. Operational research shows that length of stay is a mixed exponential function. This can be approximated by thinking in terms of half-lives rather than mean length of stay. The median length of stay is a rough but useful approximation (Priest 1995). In practice a mixed exponential function means that there will always be some patients who require much shorter and much longer lengths of stay. 


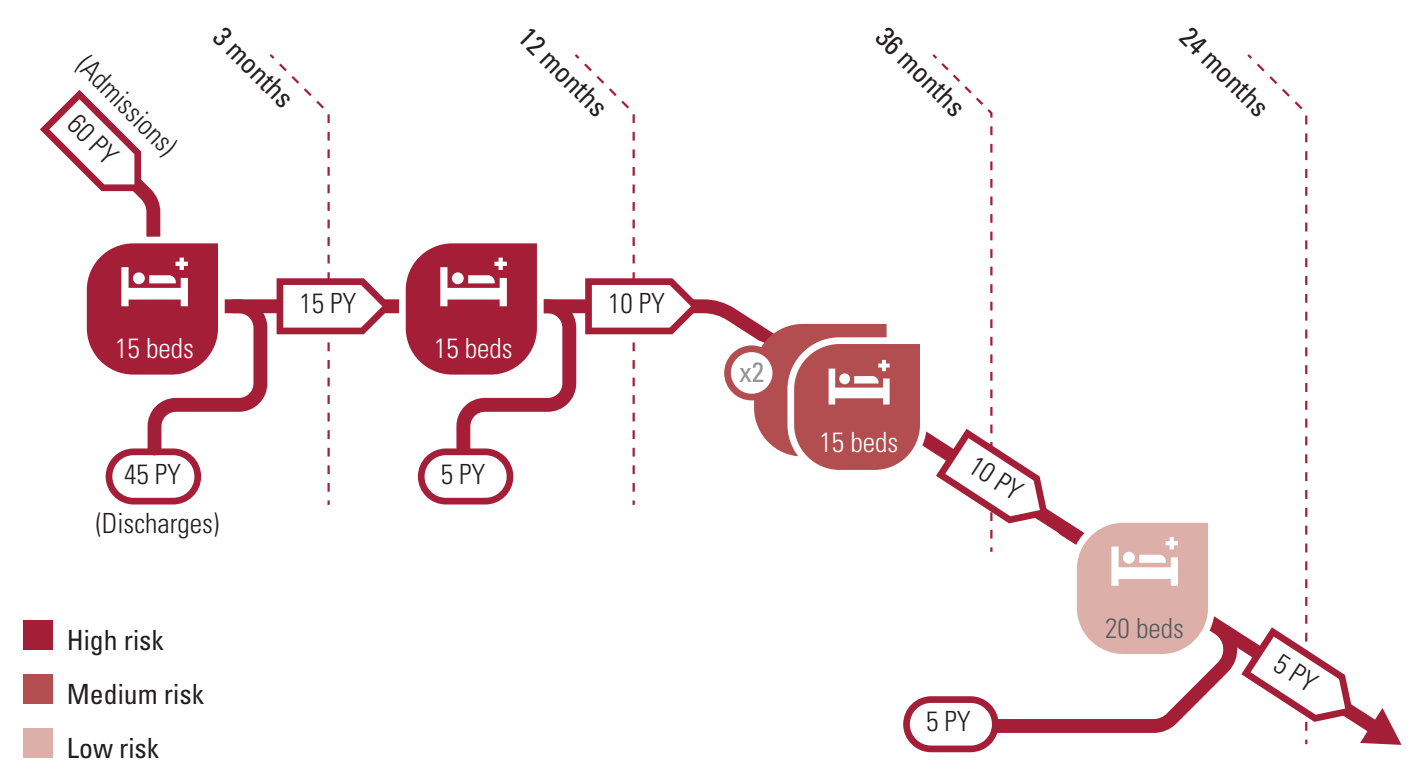

Stratified therapeutic security, the most common model of therapeutic security in secure forensic hospitals. PY, per year.

Although the majority may be discharged back to the community or the criminal justice system, unless there is provision for those requiring much longer lengths of stay, admission capacity will quickly be lost as new long-stay patients accumulate.

\section{Triage and pathways management}

Forensic psychiatrists typically act as gatekeepers for secure forensic hospitals to ensure that patients are detained in no greater or more restrictive a level of therapeutic security than is necessary and for no longer than is necessary. Structured professional judgement instruments based on validated criteria are increasingly used as decision support structures by admissions panels and other governance structures for gatekeeping and individual goal setting. This includes reports to mental health review boards. These instruments guide but do not bind the clinician and the decision maker. Such instruments should be patient focused not institution centred, rating according to the patient's characteristics and situation, not seeking to match the patient to an institutional characteristic. They should be calibrated in meaningful units of reliable change (high-, medium- or low-security needs) or difference and validated against real world operational differences (high, medium or low secure) and changes (moves between levels of care or security) (Glancy 2021; Kennedy 2019b; Shinkfield 2014).

In many jurisdictions the courts decide on committal to a forensic hospital or forensic psychiatry service on the basis of expert evidence, first regarding mental disorder, then fitness to stand trial or responsibility, then according to the need for treatment and the level of therapeutic security required. Forensic psychiatrists have an essential role in relation to each of these steps. Although the courts should be independent in the exercise of their powers, an effective secure forensic hospital service requires there to be some degree of consistency in decision-making by the courts and predictability in the numbers and triaging for admission from year to year (supplementary Box 1, available online at https://dx.doi.org/10. 1192/bja.2021.34.) (Chow 2016; Gupta 2018; NHS Digital 2017; Salize 2007). It follows that when planning a model of care, the ability to admit numbers of this order is essential, it must be sustainable over time and should include surge capacity. Modelling future needs should take account of annual admission rates and case mix (need for therapeutic security) and likely length of stay in half-lives and the effects of alternatives such as community treatment orders (Davoren 2015a; Gibbons 2015).

Admission rates and bed capacity may be driven by differences in population density, deprivation and social cohesion scores. Differences in case mix are also relevant, although the increasing use of measures of need for therapeutic security demonstrates consistent results in most high secure hospitals (Eckert 2017; Williams 2020) but not in all jurisdictions (Habets 2019). Consistency has been shown in medium secure hospitals, in prison and court triage assessments for all levels.

Specialist forensic psychiatry assessment is required to ensure appropriate admissions to secure hospitals, and this should be supported by use of validated structured professional judgement tools (Glancy 2021). Similarly, the decision to move from high to medium to low security, from acute to subacute to medium or low security and the 
community is heavily influenced by risk assessment using modern instruments for which there is objective validation, such as the 20-item Historical, Clinical and Risk Management guidelines (HCR-20) and the Dangerousness Understanding, Recovery and Urgency Manual (DUNDRUM) toolkit (Adams 2018; Davoren 2013; Wharewera-Mika 2020). A regular clinical audit of the appropriateness of admissions using a validated instrument such as the DUNDRUM-1 triage security instrument (Freestone 2015; Habets 2019; Williams 2020) would assist in ensuring sustainability.

Progress along the pathway from admission and subacute to medium secure and pre-discharge wards is based on routine assessment of risk of violence (Abidin 2013; Dolan 2010; Müller-Isberner 2007; Pillay 2008) and suicide (Abidin 2013) using structured professional judgement instruments. In intensive care, acute and subacute settings, daily short-term assessments such as the Dynamic Assessment of Situational Aggression (DASA) and the Brøset Violence Checklist (BVC) are used (Maguire 2019). Equally important is the use of repeated measures of treatment programme completion and forensic recovery (Davoren 2013, 2015b; McCullough 2020) to inform individual care planning and reports to mental health review boards.

\section{Pathways governance and management}

An admissions panel should act as gatekeeper and should then actively prioritise the waiting list for admission to the secure forensic hospital (Flynn 2011; Freestone 2015; Williams 2020).

Acute and subacute admission wards must remain capable of providing a sustainable level of admissions per year. An intensive care ward is necessary to minimise the use of seclusion, restraint and forced medication. It also prevents the disruption of the therapeutic milieu and patient safety on other wards. High staff:patient ratios and specialist skills (relational therapeutic security) are required for the prevention and management of violence and aggression, including dynamic risk assessment, de-escalation and the proportionate use of restrictive and intrusive interventions (Kennedy 2020).

Medium-term medium secure wards for those recovering from acute symptoms should orient towards psychoeducation and psychological treatments for relapse prevention, recovery from substance misuse and reducing the risk of future offending, with an emphasis on reducing also the seriousness of any future offending.

Slow-stream secure or long-term wards are necessary to accommodate those who do not benefit from interventions sufficiently to progress towards less secure or community placements. The emphasis should be on building rapport and trust while providing quality of life for self-actualisation and selftranscendence (Eckert 2017).

Pre-discharge wards provide rehabilitation for those in transition from the secure hospital to community places. The progressive use of accompanied, unaccompanied and overnight leave typically is regarded as necessary before conditional discharge. Leave is often a hazard for bringing drugs and other contraband back into the therapeutically secure environment. Limiting communication with other parts of the secure campus may be necessary at this stage. The emphasis is on occupational therapy and rehabilitation, self-medication, selfmaintenance of abstinence and relapse prevention. Family work is often critical at this or earlier stages along the pathway.

A governance structure should act as gatekeeper and pathways manager for passage between the subacute ward and the medium-term medium secure ward; and between the medium-term medium secure ward and the pre-discharge ward that leads on to the community.

\section{Specialist pathways}

Women generally make up $10-15 \%$ of patients in secure forensic hospitals, and for safety reasons require single-sex wards (Mezey 2005), although many activities can be integrated with male patients. A minimum number of wards is required to avoid mixing acutely unwell and high-risk patients with recovering and pre-discharge patients all in the one ward (Harty 2012).

A similar pathways design can be used for patients with intellectual or developmental disorders, acquired brain injury, older patients and other specialist needs.

Secure hospital provision for forensic child and adolescent patients necessitates further modifications. Special attention is required to ensure appropriate transitions from forensic child and adolescent to adult forensic psychiatry services, including processes, experience and outcomes (Livanou 2021).

\section{Active management of length of stay}

This basic model (Fig. 1) supposes that a male secure hospital pathway of about 110 beds would, subject to the governance of a medically led admissions panel and subject to interactions with the courts, admit 60 patients per year from remand or sentenced prisons, of whom 45 would have a managed length of stay of approximately 3 months before returning to the criminal justice system or stepping down via the courts to community mental health teams; the remaining 15 would spend a 
further 12 months in a subacute ward. A worked example of further active management of length of stay is given in supplementary Box 2.

\section{Community forensic residences}

Those who are on periods of trial leave in the community, or are conditionally discharged for supervision in the community, may be placed in residential settings that also have a stratified system of therapeutic security, with minimal if any physical security but varying levels of relational and procedural security. Staffing may vary from $24 \mathrm{~h}$ nurse care to social care at varying levels, with frequent contact with the supervising community forensic mental health team. Patients may progress from higher to lower levels of support and supervision over time, until they settle in independent living or find the level of support necessary to remain stable and achieve their personal optimum recovery goals in a forensic context (Adams 2018; Davoren 2015a). Patients may be reintegrated into community mental health teams at the point of transition into the community (an integrated model), remain in a forensic psychiatry service in the community (a parallel model) or reintegrate some time after transition to the community (Coid 2007; Sharma 2015).

Some alternative possible organisations of pathways and processes within secure forensic hospitals will be mentioned towards the end of this section.

\section{Variants of the standard model of care}

Much depends on the size of the population served. In many jurisdictions, forensic mental health services are organised to serve population aggregates of 3-5 million. In the provinces of Canada, the states of Australia and the Länder (federal states) of Germany this allows a single integrated service to provide high, medium and low levels of therapeutic security on a single campus, with seamless movement of patients between levels according to individual need and measured outcomes (described below as the 'four recoveries'). A critical mass can also be achieved to enable not only stratification according to levels of therapeutic security and risk, but also specialised pathways in parallel groups such as women (typically $10-15 \%$ of forensic patients), patients with intellectual and developmental disorders, patients for whom complex needs arise mainly from personality disorders, and other small specialist groups (elderly patients, patients with acquired brain injury, long-term/slow-stream patients).

Very small services (20-30 beds) may not be able to stratify (supplementary Fig. 1(a)). When patients are admitted, treated and discharged all in the same ward, this may be viable for very short-term crisis admissions but is disruptive for other patients on the ward engaged in medium-term treatments and rehabilitation. Larger hospitals or campuses may have several such wards still acting independently (supplementary Fig. 1(b)), mixing acute, subacute, medium term and pre-discharge together in each ward, with no stratification of risk or treatment and recovery stages, and no specialist wards. There may be arbitrary variation between wards according to local clinician preferences. In the alternative, a multi-ward hospital may have an admission ward, then multiple small specialist wards (supplementary Fig. 1(c)) that again mix patients at different levels of risk and different stages of treatment and recovery. The standard model of stratified therapeutic security for approximately 100 beds (supplementary Fig. 1(d)) has been described above. Services covering very large populations (15-20 million) may opt to provide highly specialised (tier 4) high-security hospitals that are separate from medium secure and low secure hospitals (serving 2-5 million) (supplementary Fig. 1(e)). Each of these may then have stratified pathways within the campus and in some cases (supplementary Fig. 1(f)) parallel pathways for special groups such as those with complex needs or neurodevelopmental disorders. This requires strong central governance (supplementary Fig. 1(g)) to ensure that patients do not suffer excessive delays when ready to move between services and between levels of therapeutic security.

These contrasting models may be compared according to criteria such as ability to match patient needs with proportionate therapeutic security levels over time, consistency for all patients, transparency of pathways, safety for patients and staff, and placement based on individual and population need (supplementary Table 1).

\section{Prison screening, court liaison and diversion}

In-reach services for remand prisons ('jails' in North America) work best when closely integrated with court liaison and diversion services for those awaiting trial (O’Neill 2016; Simpson 2018). Systematic screening of all those received at such a prison should lead seamlessly to court liaison, diversion to community mental health services or referral to a forensic hospital at an appropriate level of therapeutic security. This is an essential protection for the rights of the mentally ill, particularly when imprisonment can be related to mental health service strain at community and tier 2 level (O'Reilly 2019b). Specialist units in remand prisons may offer enhanced care while individuals are awaiting assessment or transfer to a community mental health team, tier 2 psychiatric hospital or forensic hospital. 


\section{'Sentenced prisons' and continuity of care}

For prisoners requiring psychiatric follow-up while serving their sentence (those in 'sentenced prisons'), a system of psychiatric continuity of care and monitoring is necessary. This should also work best when closely integrated with the forensic mental health service and secure forensic hospital and community services (Doyle 2014). Specialist units within prisons may offer enhanced support for those returned from periods of treatment in a forensic hospital or for those with psychiatric or psychological needs not requiring transfer to a forensic hospital.

\section{Community forensic teams}

Patients conditionally discharged following a finding of not criminally responsible who have successfully completed medium- or longer-term treatment in a secure forensic hospital are most often placed in specialist community residences with high, medium or low levels of staff support and structures. These patients are often followed both therapeutically and from the point of view of monitoring and supervision by community forensic mental health teams. These may also be integrated with probation, parole and other voluntary sector agencies. This sort of forensic mental health pathway can be described as 'parallel' to general adult and community mental health services. It is in the interests of recovery and de-stigmatisation that reintegration into mainstream services should always remain as medium- or longer-term goal.

Patients admitted to acute or subacute wards in a secure forensic hospital then diverted from the criminal justice system to community mental health services can best be thought of as following a pathway that is integrated between forensic and general adult mental health services.

\section{Continuity of care and responsibility}

A number of models for continuity of care can be compared. There may be one consultant psychiatrist-led team per ward. This pleases nurses, who have to service only one ward round a week and can expect consistency. However, consultant forensic psychiatrists and other members of the multidisciplinary team will need to have wider horizons of clinical activity to maintain their expertise, including prison, community and court work. A more common solution is to operate wards in clusters for example acute with subacute and intensive care; medium-term medium secure wards; predischarge and community. Two consultants will then follow their patients along a coherent part of the pathway, while also taking a role in the gatekeeping and transfer processes. With ward managers and senior allied health professionals, consistency can be expected in the planning of care and treatment according to individual and aggregated patient needs and treatment goals. For patients, changes of primary nurse, key worker and consultant at intervals of 1 or 2 years represent a process of learning to develop and cultivate professional and supportive relationships with appropriate boundaries (Tighe 2012) that avoid excessive personal dependence. This is essential for successful supervision in the community over periods of years.

There is much evidence that each discipline contributes qualitatively different elements to the individual care and treatment plan, but there is only opinion evidence Murphy (2002) that multidisciplinary teams in secure forensic hospitals or the community as shared decision makers contribute measurable benefit for improved risk assessment or management, shortened length of stay or improved patient satisfaction. Multidisciplinary teams impose administrative burdens on time and reduce available face-to-face time for direct patient therapeutic contact. Transdisciplinary ways of working (all members of a team carrying out the same tasks) can lead to confusion regarding boundaries, competencies and clinical responsibility.

\section{Treatments}

Ensuring that patients actually receive sufficient effective treatment is core to the purpose of a model of care (Box 4). As a tertiary referral service, forensic mental health services should be able to provide specialist treatment programmes in fulfilment of the goals of the model of care.

\section{BOX 4 Treatments, standards and staffing}

- Multimodal treatments to prevent violence and enhance recovery:

o core treatments: physical health, mental health, substance misuse, offending behaviours, family and intimacy ( $5 \mathrm{~h}$ per patient per week)

o rehabilitation: self-care and activities of daily living education occupation creativity ( $20 \mathrm{~h}$ per patient per week)

- Tiered treatments and competencies

- Quality standards:

- $25 \mathrm{~h}$ per patient per week, of which

- $5 \mathrm{~h}$ of core treatments at higher tiers per patient per week

- Logic model: inputs and outcomes

- 17.5-25 whole-time equivalent therapists for 100 patients to deliver $5 \mathrm{~h}$ core therapy for 100 patients

- Outcomes: see Box 8 
Individual care and treatment plans cannot be unrelated to the goals of the model of care for a forensic mental health service, notably violence prevention. The purpose of therapeutic security is to provide an environment in which treatments to reduce the likelihood and seriousness of future violence can be delivered safely. The primary goal of treatment may be to address causal factors for violence, including the patient's specific psychiatric problems, which often involve treatment-resistant mental illnesses and complex co-morbidities. Specialist treatments must be delivered to reduce the risk of future violence and offending and to reduce the seriousness of any future violence or adverse event (Richter 2018). Evidence of effectiveness should be grounded in randomised controlled trials (Kennedy 2019b).

Many approaches to treatment are possible. A model of care should be clear about what is intended. Reviewing delinquency and violence, McGuire (2008) concluded that the best evidence was for multimodal treatment programmes. A structural analysis suggests that secure forensic hospitals, like psychiatric services generally, deliver treatment programmes in seven domains or 'pillars'. These can be broadly classified as (a) physical health (which has gained acute importance during the current COVID-19 pandemic) (Rees 2020; Uhrskov Sørensen 2020); (b) mental health; (c) substance use disorders; (d) problem behaviours/criminogenic needs (Clarke 2011); (e) self-care and activities of daily living; (f) education occupation and creativity (O'Flynn 2018); and (g) family relationships and intimacy (Kennedy 2019a). McGuire (2008) and Wampold (2015) suggest that much greater attention should be paid to issues such as the frequency and duration of each treatment programme, broadly understandable as 'dose'. In general, each programme can be delivered in three phases: a short introductory phase that can be delivered in acute and subacute wards; a substantive phase, which may consist of multiple treatment programmes within each domain; and a maintenance, self-maintenance or 'recovery' phase, often commencing in the context of supervised or conditional discharge. Progressing from pre-contemplation and lack of insight through a cycle of change to recovery is possible only if patient choice and preference are not the only determinants of the individual treatment programme - patient choice and preference must, however, be amenable to change. Clinicians must be able to challenge beliefs and attitudes related to violence in a safe setting. In the context of preventing violence, forensic psychiatrists often must say no to requests to reduce or stop medication and must at times confront denials of problems with substance misuse or violent attitudes (Roychowdhury 2011).
Introductory phases of treatment such as psychoeducation and engagement can be delivered as tier 1 or tier 2 interventions, but substantive programmes generally require tier 3 or tier 4 skills for the therapists or for the leaders of the programme (supplementary Table 2). This enables the tailoring of treatments to the needs and neurocognitive learning style of the patient and an estimate of the likely dose-response relationship (Campbell 2011).

For example, within the pillar of mental health treatments, a patient with cognitive impairment arising from schizophrenia or a developmental disorder may commence with a cognitive remediation programme (O'Reilly 2019a). This may be followed by a programme of metacognitive therapy (Naughton 2012). These are designed to enable the patient to benefit from cognitive-behavioural therapies or other forms of intervention. Prioritising, resourcing, timetabling and accommodating for $5 \mathrm{~h}$ a week of these core therapies is a matter for the model of care, although practical details will be described in subsidiary policies and standard operating protocols.

\section{Treatment resources}

Identifying and quantifying treatment resources and activity is the first part of the logic model relating inputs to outputs (health gains). The Quality Network for Forensic Mental Health Services (QNFMHS) set as a standard that every patient should have $25 \mathrm{~h}$ a week of structured activities (Aimola 2016). In the absence of better evidence regarding the relationship between 'dose' in hours of face-to-face time with a therapist and response in terms of reduction in risk and the seriousness of risk and reduction in length of stay, a reasonable goal is to deliver $5 \mathrm{~h}$ a week of face-to-face interventions in mental health, substance misuse, problem behaviours (criminogenic need) and formal family therapy. The remaining $20 \mathrm{~h}$ should be taken up with activities of daily living and education, occupation and creativity focusing on quality of life (O’Flynn 2018), self-actualisation (Roychowdhury 2011) and self-transcendence (Wharewera-Mika 2020).

A potential failing of unwritten models of care for in-patient treatment in forensic settings is not delivering measured hours of face-to-face talking therapies by appropriately qualified therapists (Duggan 2011). A model of care must set a standard for delivery of effective treatments. The standard set must be quantified and the delivery must be quantifiable. If one whole-time therapist can deliver $20 \mathrm{~h}$ a week of face-to-face therapy with patients, then to deliver $5 \mathrm{~h}$ a week of individual face-to-face therapy for 100 patients would require 25 whole- 
time equivalents with appropriate skills. If face-toface time is made up of $3 \mathrm{~h}$ of one-to-one sessions and a $2 \mathrm{~h}$ group session involving eight patients and two therapists, this would require 17.5 wholetime equivalents for 100 patients. Further staff planning is required to ensure that therapists can deliver appropriately tiered therapies, scheduling for availability of treatment rooms and coordination with other daily and weekly routines. This should be an explicit part of the model of care.

Individual care and treatment plans are typically drafted very soon after admission and are regularly revised in the light of reassessment and progress at least every 6 months, typically before a legal review of detention. These can be used at the individual level and at the aggregated level to assess need for general and specific treatment programmes. A governance system is required to ensure that resources are systematically brought to bear to meet these treatment needs. This can take the form of a practical booking system for patients, therapists and rooms. This quantified resource, 'dose' or input is the first part of the 'logic model' of the model of care.

\section{Evaluation and logic models}

The second essential part of the model of care's 'logic model' is a process of evaluation, relating the use of resources to 'output', the achievement of health gains. Measurement-based care at the individual level is an essential aspect of evidence-based care, with recognised barriers to implementation (Glancy 2021). At the systems level, evaluating outcomes to ensure feedback is too important to go unresourced, unstructured and informal. An evaluation process is itself a marker of service quality and excellence. The goals set by the model of care comprise outcomes that must be continuously evaluated. Excellent services should have well-resourced and active teams for routine outcome evaluation and continuous feedback to clinicians, patients and review boards.

An electronic health record and clinical management system should include dashboards for continuous monitoring of therapeutic input and routine outcome measures of 'output' health gains at individual and aggregated levels. The evaluation team should monitor the quality and reliability of information fed into this system.

Three types of measure are typically taken as evaluations of the success of a model of care: health organisational performance indicators, routine outcome measures and assessment of fidelity to the model (Box 5).

Health organisational performance indicators are of interest to the commissioners of the service. These
BOX 5 Evaluation at the population/organisation and individual level

Population health and organisation performance indicators:

- sustainable admission and discharge rates over 5 years (length of stay)

- admissions per 100000 per year, discharges per 100 beds per year

- violence and restrictive practices per 100 admissions and per 100 beds

- absconding per 1000 episodes of leave outside the secure perimeter.

- positive drug screens per 100 patient-years

Individual-level indicators - the four recoveries:

- forensic recovery: consent and capacity, leave, transfer, conditional discharge, risk and protective measures; treatment programme completion; forensic recovery

- symptomatic recovery: positive, negative and general symptoms, remission rates, violent incidents and restrictive practices

- functional recovery: neurocognition and social cognition, clinical and global assessment of function, social and occupational function

- personal recovery: ward atmosphere, satisfaction, working alliance, perceived coercion; concordance of selfreported and staff-rated treatment completion and forensic recovery

indicators are usually expressed in terms of the population served, and they may be designed for accountability concerning accessibility, equitably, effectiveness, efficiency and sustainability.

Some basic performance indicators (e.g. NHS Digital 2017) are show in Table 1. Further examples might include violent and self-harming incidents per 100 admissions per year or per 100 beds per year (this may be set as a 'zero' event); escape incidents from within the secure campus per 100 patients per year and absconding from leave outside the secure campus per 1000 episodes of day leave per year. Reoffending and readmission rates are often difficult to measure in individual services but should be collated nationally and internationally. Life expectancy and standardised mortality ratios should be regarded as key outcomes for forensic as well as general mental health services for those with severe mental illness (Uhrskov Sørensen 2020).

Routine outcome measures at hospital or team level collate measurement-based care at the individual level. These typically reflect clinician- and patient-focused concerns for health gains and recovery. Routine outcome measures can be considered under four headings: forensic recovery, symptomatic recovery, functional recovery and personal recovery 
TABLE 1 Health service performance measures

\begin{tabular}{|c|c|c|c|c|}
\hline Item & Definition & Goals & Measures & Timescale \\
\hline Accessibility & Ease of access & $\begin{array}{l}\text { Admission criteria international guide: } 0.7 \text { per } \\
100000 \text { population per year (NHS Digital 2017) }\end{array}$ & Admissions per 100000 population per year & Annual \\
\hline Equitability & $\begin{array}{l}\text { Equality of access } \\
\text { Non-discrimination }\end{array}$ & & $\begin{array}{l}\text { Admissions per } 100000 \text { population by region, } \\
\text { corrected for demographics and social variables }\end{array}$ & Annual \\
\hline \multirow[t]{2}{*}{ Effectiveness } & Discharge rate & Objective return to functional autonomy and safety & Discharges per 100 beds per year & Annual \\
\hline & Moves to less secure places & Objective reduction in secure dependency needs & Positive moves per 100 beds per year & Annual \\
\hline \multirow[t]{2}{*}{ Efficiency } & Health gains per unit resource & & $\begin{array}{l}\text { Discharges per } 100 \text { beds and discharges } \\
\text { per } 100 \text { clinicians }\end{array}$ & Annual \\
\hline & & & $\begin{array}{l}\text { Moves to less secure places per } 100 \text { beds and } \\
\text { per } 100 \text { clinicians }\end{array}$ & Annual \\
\hline Sustainability & $\begin{array}{l}\text { Year-on-year levels of service } \\
\text { should not trend down }\end{array}$ & All of the above sustained over 5 -year periods & & 5-year periods \\
\hline
\end{tabular}

(Kennedy 2019a) (Box 6 and supplementary Table 3). These are broadly comparable to clinician-reported outcome measures, patient-reported outcome measures and patient-reported experience measures (Gibbons 2015).

Forensic recovery can be measured in milestones concerning increasing autonomy, independence and responsibility. Forensic recovery can be measured as changes in dynamic risk and protective factors, treatment programme completion and measures of stability, working alliance, trust and concordance (Davoren 2015b). Forensic recovery also includes the generally recognised concept of subjective recovery measured by patient-reported outcome measures and patient-reported experience measures. Ward atmosphere, subjective safety and hope are all relevant (Kennedy 2019a).

Symptomatic recovery measures are important for alleviating suffering and distress as well as managing and reducing risk of violence and suicide.

Functional recovery is increasingly recognised as one of the most important goals of treatment in psychiatry. Functional recovery is when patients achieve their greatest potential for safe and independent living, with whatever long-term supports may be needed. Reducing the vulnerability to future stress may reduce the risk of violence (Coid 2015).

Personal recovery includes measures of satisfaction, hope and working alliance.

Patient self-ratings or joint ratings of risk can be shown to be unreliable as risk predictors (Troquete 2013) and as measures of treatment completion and forensic recovery when objective outcomes are taken as validation criteria (Davoren 2015b). However, the gap between staff-rated and patient-rated measures of treatment completion and of forensic recovery (stability, insight, rapport and working alliance, use of leave, victim sensitivities, hope) has been described as a measure of concordance and this measure of concordance is predictive of objective outcomes (Davoren 2015b).
Measures of the four recoveries should be repeated at intervals of 6 months during a medium-term course of treatment in a secure forensic hospital. These should be reported to mental health review tribunals or boards as part of the treating consultant psychiatrist's structured, written report (Glancy 2021). This creates a virtuous cycle of transparency and goal setting for the patient, so that the review board hearing becomes a motivational engine for recovery or therapeutic jurisprudence. This also enables the active management of length of stay by focusing treatment resources on identified needs relevant to discharge or transfer.

An assessment of fidelity to the model (so-called implementation science) is particularly necessary for complex models of care such as a secure forensic hospital or the pathways and processes of a forensic mental health service. It is also necessary to avoid 'mission drift' in isolated parts of the service, to maintain focus on the defined goals over the planned lifetime of the model of care. Any future revision of the model of care will be informed by the evidence of the evaluations.

\section{Conclusions: quality, excellence and dynamic systems}

A written model of care is the starting point for any progressive system of delivery in forensic psychiatry. Forensic psychiatry services may to some extent be a bellwether for both positive and problematic aspects of the larger model of care for mental health services for a population and manifestations of service strain (Khosla 2014; O'Grady 1990; O'Reilly 2019b). Goals, pathways and processes, treatment systems and continuous evaluation of the logic model are essential elements. There are well-developed benchmark standards for quality of secure forensic services (Aimola 2016). Where a service falls below any of these standards, it is not too difficult to correct this. Within a network of services making up a model of care, at least some centres (usually 
the most highly specialised) should achieve excellence, with positive influences on all high-quality services in the system or network. Excellence is the process of leading continuous improvement of outcomes for patients through the virtuous cycle of research, development, teaching and training. Models of care for services that manage serious risks must ensure long-term sustainability and resilience. Investing in excellence and setting this as a key priority and value is essential (Kennedy 2019b). Multicentre research and development is now possible and should be a part of the larger model of care for a network of forensic mental health services that aspire to excellence.

\section{Supplementary material}

Supplementary material is available online at https://doi.org/10.1192/bja.2021.34.

\section{Acknowledgements}

Graphics for figures were created by Leo Kennedy. Many patients and colleagues have shaped my thinking over many years. In particular, I thank Professors Mike Doyle and Jim Ogloff, who have prompted me most in relation to this article. Professor Michael Gill and my colleagues at Trinity College Dublin, notably Professor Damian Mohan, Dr Mary Davoren and Professor Ken O'Reilly; Professor Elizabeth Urhskov-Sorensen and the University of Aarhus, Denmark; Professor Felice Caraballese of University of Bari; Professor Ingeborg Jeandarme of Louvain University; Ms Deborah Bull of NHS-England; and many others.

\section{Funding}

This research received no specific grant from any funding agency, commercial or not-for-profit sectors.

\section{Declaration of interest}

None.

\section{References}

Abidin Z, Davoren M, Naughton L, et al (2013) Susceptibility (risk and protective) factors for in-patient violence and self-harm: prospective study of structured professional judgement instruments START and SAPROF, DUNDRUM-3 and DUNDRUM-4 in forensic mental health services. BMC Psychiatry, 13: 197

Adams J, Thomas SD, Mackinnon T, et al (2018) The risks, needs and stages of recovery of a complete forensic patient cohort in an Australian state. BMC Psychiatry, 18: 35.

Agency for Clinical Innovation (2013) Understanding the Process to Develop a Model of Care: An ACl Framework (Version 1.0, May 2013). NSW Agency for Clinical Innovation.
Aimola L, Jasim S, Tripathi N, et al (2016) Impact of peer-led quality improvement networks on quality of inpatient mental health care: study protocol for a cluster randomized controlled trial. BMC Psychiatry, 16: 331.

Campbell M (2011) The Matrix: A Guide to Delivering Evidence-based Psychological Therapies in Scotland (The Matrix 2011). The Scottish Government.

Chow WS, Priebe S (2016) How has the extent of institutional mental healthcare changed in Western Europe? Analysis of data since 1990. BMJ Open, 29(6): e010188

Clarke PDR, Gilchrist L, Johnstone L, et al (2011) Chapter 6: The Matrix Evidence tables - summary of the psychological therapies evidence base, forensic mental health section. In The Matrix: A Guide to Delivering Evidence-Based Psychological Therapies in Scotland (The Matrix 2011) (ed M Campbell): 241-249. The Scottish Government.

Coid JW, Hickey N, Yang M (2007) Comparison of outcomes following after-care from forensic and general adult psychiatric services. British Journal of Psychiatry, 190: 509-14.

Coid JW, Kallis C, Doyle M, et al (2015) Identifying causal risk factors for violence among discharged patients. PLoS One, 10: e0142493.

Davoren M, Abidin Z, Naughton L, et al (2013) Prospective study of factors influencing conditional discharge from a forensic hospital: the DUNDRUM-3 programme completion and DUNDRUM-4 recovery structured professional judgement instruments and risk. BMC Psychiatry, 13: 185.

Davoren M, Byrne 0, O'Connell P, et al (2015a) Factors affecting length of stay in forensic hospital setting: need for therapeutic security and course of admission. BMC Psychiatry, 15: 301

Davoren M, Hennessy S, Conway C, et al (2015b) Recovery and concordance in a secure forensic psychiatry hospital: the self rated DUNDRUM-3 programme completion and DUNDRUM-4 recovery scales. BMC Psychiatry, 15: 61

Denyer D (2017) Organizational Resilience: A Summary of Academic Evidence, Business Insights and New Thinking. BSI and Cranfield School of Management.

Dolan M, Blattner R (2010) The utility of the Historical Clinical Risk-20 Scale as a predictor of outcomes in decisions to transfer patients from high to lower levels of security-A UK perspective. BMC Psychiatry, 10: 76.

Doyle M, Coid J, Archer-Power L, et al (2014) Discharges to prison from medium secure psychiatric units in England and Wales. British Journal of Psychiatry, 205: 177-82.

Duggan C (2011) Dangerous and severe personality disorder. British Journal of Psychiatry, 198: 431-433.

Eckert M, Schel SH, Kennedy HG, et al (2017) Patient characteristics related to length of stay in Dutch forensic psychiatric care. Journal of Forensic Psychiatry \& Psychology, 28: 863-80

Falk M (1985) Secure facilities in local psychiatric hospitals. In Secure Provision (ed LO Gostin). Tavistock Publications.

Flynn G, O'Neill C, Kennedy HG (2011) DUNDRUM-2: prospective validation of a structured professional judgment instrument assessing priority for admission from the waiting list for a forensic mental health hospital. BMC Research Notes, 4: 230.

Freestone M, Bull D, Brown R, et al (2015) Triage, decision-making and follow-up of patients referred to a UK forensic service: validation of the DUNDRUM toolkit. BMC Psychiatry, 15: 239

Gibbons L, McCarthy L (2015) Payment by results in forensic mental health. BJPsych Bulletin, 39: 209-12.

Giblin Y, Kelly A, Kelly E, et al (2012) Reducing the use of seclusion for mental disorder in a prison: implementing a high support unit in a prison using participant action research. International Journal of Mental Health Systems, 6(1): 2.

Glancy G, Choptiany M, Jones R, et al (2021) Measurement-based care in forensic psychiatry. International Journal of Law and Psychiatry, 74: 101650

Gunn J (1977) Management of the mentally abnormal offender: integrated or parallel. Proceedings of the Royal Society of Medicine, 70: 877-80.
MCQ answers

$1 d \quad 2 b \quad 3 b \quad 4 b \quad 5 a$ 
Gupta S, Akyuz EU, Baldwin T, et al (2018) Community treatment orders in England: review of usage from national data. BJPsych Bulletin, 42(3): 119-22.

Habets P, Jeandarme I, Kennedy HG (2019) Applicability of the DUNDRUM-1 in a forensic Belgium setting. Journal of Forensic Practice, 21: 85-94.

Harty M, Somers N, Bartlett A (2012) Women's secure hospital services: national bed numbers and distribution. Journal of Forensic Psychiatry \& Psychology, 23: 590-600.

James DV, Fineberg NA, Shah AK, et al (1990) An increase in violence on an acute psychiatric ward. A study of associated factors. British Journal of Psychiatry, 156: 846-52.

Kennedy $\mathrm{H}$ (2002) Therapeutic uses of security: mapping forensic mental health services by stratifying risk. Advances in Psychiatric Treatment, 8 : 433-43.

Kennedy HG, Simpson A, Haque 0 (2019a) Perspective on excellence in forensic mental health services: what we can learn from oncology and other medical services. Frontiers in Psychiatry, 10: 733.

Kennedy HG, O'Reilly K, Davoren M, et al (2019b) How to measure progress in forensic care. In Long-Term Forensic Psychiatric Care (eds B Völlm, P Braun). Springer.

Kennedy HG, Mullaney R, McKenna P, et al (2020) A tool to evaluate proportionality and necessity in the use of restrictive practices in forensic mental health settings: the DRILL tool (Dundrum Restriction, Intrusion and Liberty Ladders). BMC Psychiatry, 20: 515

Khosla V, Davison P, Gordon H, et al (2014) The interface between general and forensic psychiatry: the present day. Advances in Psychiatric Treatment, 20: 359-65.

Lemieux AJ, Dumais Michaud A-A, Damasse J, et al (2020) Management of COVID-19 for persons with mental illness in secure units: a rapid international review to inform practice in Québec. Victims \& Offenders, 15: 1337-60

Livanou M, D'Souza S, Lane R, et al (2021) Challenges and facilitators during transitions from adolescent medium secure units to adult services in England: interviews with mental healthcare professionals. Administration and Policy in Mental Health [Epub ahead of print] 24 Feb. Available from: https://doi.org/10.1007/s10488-021-01115-9.

Maguire T, Daffern M, Bowe SJ, et al (2019) Evaluating the impact of an electronic application of the Dynamic Appraisal of Situational Aggression with an embedded Aggression Prevention Protocol on aggression and restrictive interventions on a forensic mental health unit. International Journal of Mental Health Nursing, 28: 1186-97.

McCullough S, Stanley C, Smith H, et al (2020) Outcome measures of risk and recovery in Broadmoor High Secure Forensic Hospital: stratification of care pathways and moves to medium secure hospitals. BJPsych Open, 6 e74.

McGuire J (2008) A review of effective interventions for reducing aggression and violence. Philosophical Transactions of the Royal Society of London. Series B, Biological Sciences, 363: 2577-97.

Mezey G, Hassell Y, Bartlett A (2005) Safety of women in mixed-sex and single-sex medium secure units: staff and patient perceptions. British Journal of Psychiatry, 187: 579-82.

Müller-Isberner R, Webster CD, Gretenkord L (2007) Measuring progress in hospital order treatment: relationship between levels of security and $C$ and R scores of the HCR-20. International Journal of Forensic Mental Health, 6: 113-21.

Murphy D (2002) Risk assessment as collective clinical judgement. Criminal Behaviour and Mental Health, 12(2): 169-78.

Naughton M, Nulty A, Abidin Z, et al (2012) Effects of group metacognitive training (MCT) on mental capacity and functioning in patients with psychosis in a secure forensic psychiatric hospital: a prospective-cohort waiting list controlled study. BMC Research Notes, 5: 302.

NHS Digital (2017) Mental Health Act Statistics, Annual Figures: 2016 17, Experimental statistics. NHS Digital (https://digital.nhs.uk/data-andinformation/publications/statistical/mental-health-act-statistics-annualfigures/mental-health-act-statistics-annual-figures-2016-17-experimental-statistics [cited 11 May 2021])
O'Flynn P, O'Regan R, O'Reilly K, et al (2018) Predictors of quality of life among inpatients in forensic mental health: implications for occupational therapists. BMC Psychiatry, 18: 16

O'Grady J (1990) The complementary roles of regional and local secure provision for psychiatric patients. Health Trends, 22: 14-16.

O'Neill C, Smith D, Caddow M, et al (2016) STRESS-testing clinical activity and outcomes for a combined prison in-reach and court liaison service: a 3-year observational study of 6177 consecutive male remands. International Journal of Mental Health Systems, 10: 67.

O'Reilly R, Allison S, Bastiampiallai T (2019a) Observed outcomes: an approach to calculate the optimum number of psychiatric beds. Administration and Policy in Mental Health, 46: 507-17.

O'Reilly K, Donohoe G, O'Sullivan D, et al (2019b) A randomized controlled trial of cognitive remediation for a national cohort of forensic patients with schizophrenia or schizoaffective disorder. BMC Psychiatry, 19: 27.

Pillay SM, Oliver B, Butler L, et al (2008) Risk stratification and the care pathway. Irish Journal of Psychological Medicine, 25: 123-7.

Priest RG, Fineberg N, Merson S, et al (1995) Length of stay of acute psychiatric inpatients: an exponential model. Acta Psychiatrica Scandinavica, 92: 315-7.

Rees C, Thomson L (2020) Exploration of morbidity, suicide and all-cause mortality in a Scottish forensic cohort over 20 years. BJPsych Open, 6 : e62.

Richter MS, O'Reilly K, O'Sullivan D, et al (2018) Prospective observational cohort study of 'treatment as usual' over four years for patients with schizophrenia in a national forensic hospital. BMC Psychiatry, 18: 289.

Roychowdhury A (2011) Bridging the gap between risk and recovery: a human needs approach. The Psychiatrist, 35: 68-73.

Salize HJ, Dressing H (2007) Admission of mentally disordered offenders to specialized forensic care in fifteen European Union member states. Social Psychiatry and Psychiatric Epidemiology, 42(4): 326-42.

Seppanen A, Tormanen I, Shaw C, et al (2018) Modern forensic psychiatric hospital design: clinical, legal and structural aspects. International Journal of Mental Health Systems, 12: 58.

Sharma A, Dunn W, O'Toole C, et al (2015) The virtual institution: crosssectional length of stay in general adult and forensic psychiatry beds. International Journal of Mental Health Systems, 9: 25.

Shinkfield G, Ogloff J (2014) A review and analysis of routine outcome measures for forensic mental health services. International Journal of Forensic Mental Health, 13: 252-71.

Simpson AIF, Jones RM (2018) Two challenges affecting access to care for inmates with serious mental illness: detecting illness and acceptable services. Canadian Journal of Psychiatry, 63: 648-50.

Tighe J, Gudjonsson GH (2012) See, Think, Act Scale: preliminary development and validation of a measure of relational security in mediumand low-secure units. Journal of Forensic Psychiatry \& Psychology, 23: 184-99.

Troquete NAC, van den Brink RHS, Beintema H, et al (2013) Risk assessment and shared care planning in out-patient forensic psychiatry: cluster randomised controlled trial. British Journal of Psychiatry, 202(5): 365-71.

Uhrskov Sørensen L, Bengtson S, Lund J, et al (2020) Mortality among male forensic and non-forensic psychiatric patients: matched cohort study of rates, predictors and causes-of-death. Nordic Journal of Psychiatry, 74: 489-96.

Von Bertalanffy L (1969) General system theory: Foundations, development, applications. George Braziller, Inc. New York.

Wampold BE (2015) How important are the common factors in psychotherapy? An update. World Psychiatry, 14: 270-7.

Wharewera-Mika J, Cooper E, Wiki N, et al (2020) The appropriateness of DUNDRUM-3 and DUNDRUM-4 for Māori in forensic mental health services in New Zealand: participatory action research. BMC Psychiatry, 20: 61 .

Williams HK, Senanayke M, Ross CC, et al (2020) Security needs among patients referred for high secure care in Broadmoor Hospital England. BJPsych Open, 6: e55. 
Multiple choice questions

Select the single best option for each question stem

1 A model of care for a forensic mental health service:

a is not influenced by other health, social care and criminal justice services

b is unique to each service, with no common features

c is a statement of principles, values and aspirations

$\mathrm{d}$ broadly defines how a health service is delivered to ensure that patients get the right care at the right time by the right team and in the right place

e should be generated spontaneously from focus groups and qualitative interviews with patients.

\section{Goals for a forensic psychiatry service} include:

a prioritising good ideas

b appropriate physical security, high staff:patient ratios and a therapeutic policy that encompasses individual programmes

c limiting violence to acceptable levels in the hospital

d prioritising patient choices over treating the causes of violence

e providing care and treatment regardless of the length of stay.
3 As regards pathways and processes in a forensic model of care:

a prison in-reach clinics and court liaison and diversion schemes are not part of the forensic model of care

b triage criteria should match the patient to the level of care and therapeutic security they need for safe treatment

c qualitative relational therapeutic security should ensure that no fixed boundaries or rules exist in the therapeutic relationship

d quantitative relational therapeutic security should ensure constant staff:patient ratios in all settings

e therapeutic risks are always beneficial and need not balance the risk of adverse outcomes.

4 As regards treatment programmes to reduce violence proneness in forensic mental health services:

a all forms of treatment are equally ineffective

b multimodal treatment is supported by the best evidence for effectiveness

c frequency and duration of treatment programmes is irrelevant to outcome

d randomised controlled trials are not possible

e patient choice and preference are the only determinants of treatment programmes in forensic mental health.
5 As regards evaluation of the model of care in forensic mental health:

a it should measure treatments delivered and routine outcomes for health and therapeutic gains

b disclosure of serial routine outcome measures to review boards is best avoided

c evaluation should be omitted from the model of care in small, short-term or unique forensic mental health services

d models of care in different jurisdictions cannot be compared by means of population-based performance indicators or routine outcome measures

e models of care cannot be improved through processes of evaluation, research and development. 